Received: 2014.08.17

Accepted: 2014.09.05

Published: 2014.09.15

\title{
The Incidence and Risk Factors of Severe Retinopathy of Prematurity in Extremely Low Birth Weight Infants in Turkey
}

Authors' Contribution: Study Design A Data Collection B Statistical Analysis C Data Interpretation D Manuscript Preparation E Literature Search F Funds Collection $G$
ABCDEF 1,2 Ali Riza Cenk Celebi

AB 1 Ikbal Seza Petricli

$A B \quad 1$ Emre Hekimoglu

A 3 Nihal Demirel

A 3 Ahmet Yagmur Bas
1 Department of Ophthalmology, Etlik Zubeyde Hanim Maternity and Women's Health Research Hospital, Ankara, Turkey

2 Department of Ophthalmology, Nigde State Hospital, Nigde, Turkey 3 Neonatal Intensive Care Unit, Department of Neonatology, Etlik Zübeyde Hanim Women's Health Teaching and Research Hospital, Ankara, Turkey
Corresponding Author: Source of support:
Ali Riza Cenk Celebi, e-mail: arcenkcelebi@gmail.com Departmental sources

Background: The aim of this study was to identify the incidence of severe retinopathy of prematurity (ROP) requiring laser treatment and the overall incidence of ROP in extremely low birth weight (ELBW) infants, and to evaluate the associated risk factors for developing severe ROP in ELBW infants in Turkey.

Material/Methods: This retrospective, case-control, tertiary care-hospital based study included 235 ELBW infants screened for ROP. The incidence of the onset of ROP and severe ROP requiring laser treatment were assessed. The relationship between various clinical risk factors, and the development of severe ROP and onset of ROP were analyzed using univariate analysis and multivariate logistic regression analysis.

Results: The overall incidence of any stage ROP and severe ROP requiring laser treatment in ELBW infants was $75.5 \%$ and $38.7 \%$, respectively. Severe ROP requiring laser treatment in ELBW infants was significantly associated with various independent risk factors, including blood transfusion $(P=0.002)$, gestational age at birth $(P<0.001)$, gestational weight at birth $(P=0.001)$, culture-proven sepsis $(P=0.047)$.

Conclusions: The severity of ROP may increase as weight at birth and gestational age at birth decrease, and in those with culture-proven sepsis as well as in those that receive blood transfusion. Clinicians should be aware of the presence of these risk factors when treating ELBW premature infants. Early detection and prevention of sepsis and reducing the number of blood transfusions may decrease the incidence of severe ROP requiring laser treatment.

MeSH Keywords: Gestational Age • Infant, Extremely Premature • Prevalence • Retinopathy of Prematurity • Risk Factors • Turkey

Full-text PDF: http://www.medscimonit.com/abstract/index/idArt/892262

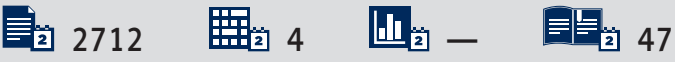




\section{Background}

Retinopathy of prematurity (ROP) is a vasoproliferative disease of the retina that affects preterm neonates and is thought to be mediated by alterations in insulin-like growth factor-1 (IGF1) and vascular endothelial growth factor (VEGF) [1]. ROP is one of the major causes of avoidable childhood blindness in developed and developing countries. Approximately 50000 children worldwide have ROP-related blindness [2]. The increase in survival rates in extremely low birth weight (ELBW) infants due to improvements in neonatal intensive care has resulted in an increased risk of developing ROP [3]. However, some studies report that improvements in neonatal intensive care have resulted in a decrease in the incidence of ROP $[4,5]$. Nonetheless, it is known that ROP develops most frequently and most severely in ELBW infants [6].

Recent studies report many systemic risk factors (respiratory distress syndrome, sepsis, necrotizing enterocolitis, intraventricular hemorrhage, and blood transfusion for neonatal severe anemia) associated with ROP [7-9]. The probable cause of the severity of ROP in ELBW infants could be associated with the risk factors stated above. The aim of the present study was to determine the incidence of severe ROP requiring laser treatment and the overall incidence of ROP, and to evaluate the risk factors for developing severe ROP requiring laser treatment in ELBW infants in Turkey.

\section{Material and Methods}

This retrospective, case-control study included preterm infants that presented to the ROP Screening, Treatment, and Training Center, Department of Ophthalmology, Etlik Zubeyde Hanim Maternity and Women's Health Research Hospital, Turkey, between January 2010 and April 2013. This study followed the tenets of the Declaration of Helsinki. The study protocol was approved by the Etlik Zubeyde Hanim Maternity and Women's Health Research Hospital Institutional Review Board. All infants with a gestational age of $<32$ week at birth were screened for ROP. The first examination was at a postnatal age of 4 weeks, but in the most immature infants (gestational age <27 weeks), the first examination was postponed until a postnatal age of 31 weeks. All preterm infants with a birth weight $\leq 1000$ g were included in this study. All associated systemic findings; including blood culture-proven sepsis, respiratory distress syndrome (RDS), necrotizing enterocolitis (NEC), intraventricular hemorrhage (IVH), blood product transfusion, and patent ductus arteriosus (PDA) were recorded.

Pupils were dilated using 2 drops of cyclopentolate/tropicamide $0.5 \%$ and phenylephrine $2.5 \%$ at 10 -min intervals. After pupils were dilated, ophthalmological examination was performed using a pediatric eye speculum and pediatric scleral depressor, following instillation of $0.5 \%$ proparacaine eye drops for topical anesthesia. Indirect ophthalmoscopy was performed using a binocular indirect ophthalmoscope with a 20 and/or 28 diopter lens. The ROP status of each infant was classified according to the International Classification of ROP, including stage, zone, and extent of disease, and presence or absence of plus disease [10]. Infants with ROP were routinely re-examined every few days to 2 weeks, depending of the zone and severity of ROP [11]. Infants requiring laser photocoagulation treatment were identified based on Early Treatment for Retinopathy of Prematurity Randomized Trial criteria [12]. Accordingly, patients with type 1 ROP (severe ROP), defined as zone 1 any stage ROP with plus disease, zone 1 stage 3 ROP without plus disease, and zone 2 stage 2 or 3 ROP with plus disease, were referred for treatment. A wait-and-watch policy was used for type 2 ROP defined as zone 1 stage 1 or 2 ROP without plus disease, and zone 2 stage 3 ROP without plus disease. Patients diagnosed with type 1 disease were immediately referred for laser photocoagulation (Iridex Oculight SL/SLX $810 \mathrm{~nm}$ diode laser, Mountain View, CA, USA) treatment the same day. The highest stage of ROP during followup examination was recorded for each infant. The other variables recorded were birth weight (BW), gestational age (GA), sex, presence of RDS, presence of blood culture-proven sepsis, presence of NEC, presence of IVH, and presence of PDA. These variables were recorded to compare screened infants that had ROP and those that did not. The patients with ROP were further divided into 2 subgroups: ROP cases not requiring laser treatment and ROP cases that received laser treatment. The variables were compared between the 3 groups (infants without ROP, infants with ROP that did not require laser treatment, and infants with ROP that received laser treatment).

\section{Statistical analysis}

Statistical analysis was performed using SPSS v.16.0 for Windows (SPSS, Inc. Chicago, IL, USA). Continuous variables are presented as mean $\pm S D$; categorical variables are expressed as number and percentage. The Kolmogorov-Smirnov test was used to analyze the normality of data distribution. Descriptive statistics, such as frequencies of NEC, PDA, IVH, blood transfusion, RDS, and sepsis, were analyzed. Mean gestational weight and gestational age at birth were compared between groups using Kruskal-Wallis analysis. The chi-square test was used to compare categorical data related to clinical outcome. Univariate analysis was conducted to assess other potential risk factors for stage 3 ROP requiring laser treatment, such as sex, sepsis, blood transfusions, IVH, PDA, and NEC. To control for all of the variables and estimate the independent significant risk factors associated with stage 3 ROP requiring laser treatment, the factors were entered into a logistic regression multivariate analysis using a stepwise model. The level of 
statistical significance was set at $P<0.05$. The adjusted odds ratio (OR) and $95 \%$ confidence interval $(\mathrm{Cl})$ for each possible risk factor were calculated.

\section{Results}

In total, 235 infants weighing $<1000 \mathrm{~g}$ were included in the study. Mean birth weight and mean gestational age were $858.31 \pm 107.45 \mathrm{~g}$ (range: $570-1000 \mathrm{~g}$ ) and $27.08 \pm 1.99$ weeks (range: 23-33 weeks), respectively. In all, 129 of the infants were female (54.9\%) and 106 were male (45.1\%). Among the patients, 48 (20.4\%) developed culture-proven sepsis, 147 (62.5\%) received surfactant and/or required oxygen therapy due to RDS, 12 (5.1\%) developed IVH, 11 (4.7\%) had NEC, 54 (22.9\%) developed PDA, and 53 (22.5\%) received blood transfusions. All baseline characteristics and associated systemic findings in the study population are summarized in Table1.

In overall, 62 (26.4\%) patients did not develop any stage of ROP. Mean birth weight and gestational age in the infants without ROP were $915.56 \pm 81.27 \mathrm{~g}$ (range: $600-1000 \mathrm{~g}$ ) and $28.34 \pm 1.91$ weeks (range: 25-33 weeks), respectively; the male-to-female ratio was $0.82(28 / 34)$.

Among the patients developing any stage of ROP, $37.02 \%$ $(n=87)$ did not require laser treatment. In total, 61 (25.9\%) patients developed stage 1 ROP and 26 (11.06\%) developed stage 2 ROP. Mean birth weight and gestational age in the ROP patients that did not require laser treatment were $862.87 \pm 101.24$ $\mathrm{g}$ (range: $600-1000 \mathrm{~g}$ ) and $27.06 \pm 1.96$ weeks (range: $23-33$ ), respectively, and the male-to-female ratio was 0.64 (34/53).

There were 86 patients (36.6\%) that received laser treatment due to severe ROP or type 1 ROP. Mean birth weight and gestational age in these patients were $816.33 \pm 110.68 \mathrm{~g}$ (range: $570-1000 \mathrm{~g}$ ) and $26.28 \pm 1.65$ weeks (range: $23-32$ ), respectively, and the male-to-female ratio was 1.04 (44/42). There were 21 patients with aggressive posterior ROP, of which 3 had diode laser treatment and progressed to stage 4 ROP. These 3 patients had vitreoretinal surgery. Other laser-treated eyes with signs of severe ROP showed disease regression during the first week and the regression was almost complete at 1 month after the laser therapy. The differences between number of ROP distributions, mean gestational weight, and mean gestational age at birth in the 3 groups were statistically significant (KruskallWallis Analysis $\mathrm{P}<0.05)$. The number and percentage of patients without ROP, and those with ROP not requiring and received laser, and their gender distribution and associated systemic findings are shown in Table 2. Table 3 summarizes the mean gestational age and mean gestational weight in the patients without ROP, those with ROP not requiring and requiring laser treatment, and the entire study population.
Table 1. Baseline characteristics and associated systemic findings in the study population.

\begin{tabular}{|ccc}
\hline Baseline Characteristics & & \\
\hline Gender & $n$ & $(\%)$ \\
\hline Male & 106 & $(45.1)$ \\
\hline Female & 129 & $(54.9)$ \\
\hline Mean \pm SD gestational age at birth (weeks) & $27.08 \pm 1.99$ \\
\hline Mean \pm SD gestational weight at birth (g) & $858.31 \pm 107.45$ \\
\hline Associated systemic findings $\mathrm{n}(\%)$ & & \\
\hline RDS & 147 & $(62.5)$ \\
\hline PDA & 54 & $(22.9)$ \\
\hline Blood transfusion & 53 & $(22.5)$ \\
\hline Sepsis & 48 & $(20.4)$ \\
\hline IVH & 12 & $(5.1)$ \\
\hline NEC & 11 & $(4.7)$ \\
\hline
\end{tabular}

According to univariate analysis, the only significant risk factor for the presence of any stage ROP in ELBW infants was gestational age at birth $(\mathrm{P}=0.046)$, but the main risk factors for ROP requiring laser treatment were low gestational age at birth $(P=0.008)$, low gestational weight at birth $(P=0.011)$, culture-proven sepsis $(P=0.017)$, and blood product transfusion $(P=0.0001)$. The other associated systemic findings, including the presence of NEC $(P=0.38)$, presence of PDA $(P=0.33)$, and presence of RDS $(P=0.35)$ and any stage IVH $(P=0.43)$, were not associated with severe ROP. Univariate analysis showed that there was a significant relationship between ROP requiring laser treatment, and low gestational age, low gestational weight, sepsis, and blood transfusions. These risk factors were entered into multivariate logistic regression analysis via the stepwise (likelihood ratio) method, in which these 4 variables (low gestational age, low gestational weight, sepsis, and blood transfusions) were identified as weak (according to low ORs) but statistically significant $(p<0.05)$ independent risk factors for severe ROP according to the $P$ values and correlation based on adjusted ORs (Table 4). A strong association was observed between blood transfusion and severity of ROP (adjusted OR: 2.165). Gestational age at birth (adjusted OR: 1.410; 95\% $\mathrm{Cl}$ : 1.175-1.694), gestational weight at birth (adjusted OR: 1.115; 95\% Cl: 1.112-1.118), culture-proven sepsis (adjusted OR: 0.464; 95\% Cl: 0.218-0.989) were weak (due to low ORs) but statistically significant $(p<0.05)$ factors predictive of severe ROP (Table 4).

\section{Discussion}

According to earlier studies, the number of extremely premature infants that survive has been increasing as neonatal care 
Table 2. The number and percentage of patients without ROP, and those with ROP not requiring laser and requiring laser, and the gender distribution, and associated systemic findings.

\begin{tabular}{|c|c|c|c|c|c|c|c|}
\hline \multirow{2}{*}{ Gender } & \multicolumn{6}{|c|}{ ROP STATUS } & \multirow[b]{2}{*}{ Total } \\
\hline & \multicolumn{2}{|c|}{$\begin{array}{c}\text { ROP negative } \\
n(\%)\end{array}$} & \multicolumn{2}{|c|}{$\begin{array}{c}\text { Non-laser-treated ROP } \\
\text { n (\%) }\end{array}$} & \multicolumn{2}{|c|}{$\begin{array}{l}\text { Laser-treated ROP } \\
\text { n (\%) }\end{array}$} & \\
\hline Male & 28 & $(26.41)$ & 34 & (32.07) & 44 & $(41.51)$ & 106 \\
\hline Female & 34 & $(26.35)$ & 53 & (41.08) & 42 & $(32.56)$ & 129 \\
\hline \multicolumn{8}{|l|}{ RDS } \\
\hline Present & 34 & (23.13) & 60 & $(40.82)$ & 53 & $(36.05)$ & 147 \\
\hline Not present & 28 & (31.81) & 27 & $(30.68)$ & 33 & $(37.50)$ & 88 \\
\hline \multicolumn{8}{|l|}{ Sepsis } \\
\hline Present & 5 & $(10.42)$ & 20 & $(41.67)$ & 23 & $(47.92)$ & 48 \\
\hline Not present & 57 & (30.48) & 67 & $(35.83)$ & 63 & $(33.69)$ & 187 \\
\hline \multicolumn{8}{|l|}{ NEC } \\
\hline Present & 1 & (9.09) & 4 & (36.36) & 6 & $(54.55)$ & 11 \\
\hline Not present & 61 & $(27.23)$ & 84 & $(37.50)$ & 79 & $(35.27)$ & 224 \\
\hline \multicolumn{8}{|l|}{ IVH } \\
\hline Present & 3 & $(25.00)$ & 4 & $(33.33)$ & 5 & $(41.67)$ & 12 \\
\hline Not present & 59 & $(26.46)$ & 78 & (34.98) & 90 & $(40.36)$ & 223 \\
\hline \multicolumn{8}{|l|}{ PDA } \\
\hline Present & 8 & $(14.81)$ & 30 & $(55.56)$ & 16 & $(29.63)$ & 54 \\
\hline Not present & 54 & $(29.83)$ & 57 & $(31.49)$ & 70 & $(38.67)$ & 181 \\
\hline \multicolumn{8}{|c|}{ Blood transfusion } \\
\hline Present & 3 & $(5.66)$ & 22 & $(41.51)$ & 28 & $(52.83)$ & 53 \\
\hline Not present & 59 & $(32.42)$ & 59 & $(32.42)$ & 64 & $(35.16)$ & 182 \\
\hline
\end{tabular}

Table 3. Mean gestational age and mean gestational weight in the patients without ROP, those with ROP not requiring and requiring laser treatment, and the entire study population.

\begin{tabular}{lcccc} 
& & ROP status & & $\begin{array}{c}\text { Overall group } \\
\text { (Mean } \pm \text { SE) }\end{array}$ \\
\hline Mean \pm SD gestational age & $28.34 \pm 1.91$ & Non-laser ROP & Laser treated ROP & $27.08 \pm 1.99$ \\
\hline Mean \pm SD birth weight & $915.56 \pm 81.27$ & $862.87 \pm 101.24$ & $816.33 \pm 110.68$ & $858.31 \pm 107.46$ \\
\hline
\end{tabular}

improves [13-16]. It has also been reported that extremely premature infants have a higher risk of developing severe ROP requiring treatment than do more mature infants [8,17-19]. The Early Treatment for Retinopathy of Prematurity (ETROP) study reported that the incidence of ROP was $82.5 \%$ in infants with a birth weight $<1000 \mathrm{~g}$, and that treatment was required in $92.4 \%$ of infants that weighed $<1000 \mathrm{~g}$ [15]. According to the Cryotherapy for Retinopathy of Prematurity (CRYO-ROP) study, $\geq 81.6 \%$ infants that weighed $<1000 \mathrm{~g}$ at birth developed ROP [20]. Kumar et al. reported that $32.8 \%$ of infants 
Table 4. Independent factors associated with ROP onset and progression to severe ROP requiring laser treatment in ELBW infants, according to multivariate logistic regression analysis.

\begin{tabular}{|c|c|c|c|}
\hline & Adjusted OR & $95 \% \mathrm{Cl}$ & $\mathbf{P}$ \\
\hline \multicolumn{4}{|l|}{ For onset of ROP } \\
\hline Gestational age at birth & 1.558 & $1.321-1.835$ & $<0.001$ \\
\hline \multicolumn{4}{|l|}{ For progression to severe ROP } \\
\hline Blood transfusion & 2.165 & $1.332-3.509$ & 0.002 \\
\hline Gestational age at birth & 1.410 & $1.175-1.694$ & $<0.001$ \\
\hline Gestational weight at birth & 1.115 & $1.112-1.118$ & 0.001 \\
\hline Presence of culture-proven sepsis & 0.464 & $0.218-0.989$ & 0.047 \\
\hline
\end{tabular}

with a birth weight $<1000 \mathrm{~g}$ had ROP [21]. Lorenz et al. noted ROP in $62.1 \%$ of infants with a birth weight $<1000 \mathrm{~g}$ [22]. A multicenter study by Zin et al. reported that $61.9 \%$ of infants with a birth weight $<1000 \mathrm{~g}$ had severe ROP [23] and Choo et al. found that $58.6 \%$ of infants weighing $<1000 \mathrm{~g}$ at birth developed ROP [24]. A recent study from China reported $64 \%$ of infants with a birth weight $<1000 \mathrm{~g}$ developed any stage ROP [25]. In the present study, $73.6 \%$ of infants had ROP, which is similar to the rates reported by other Turkish referral centers of $70.7 \%$ [26] and $75.6 \%$ [27], but lower than that in extremely premature Japanese infants (86.1\%) [28].

The rate of type 1 ROP requiring laser treatment among 235 ELBW infants in the present study was $36.6 \%$; only 1 study (by Hiraoka et al.) reported a higher percentage of ELBW infants with severe ROP requiring laser treatment (41\%) [28]. The incidence of type 1 ROP in ELBW infants was reported as $36.4 \%$ by Gharaibeh et al. [29] and Wani et al. [30], which is similar to the rate in the present study. Choo et al. reported that $32.9 \%$ of ELBW infants reached a stage of ROP requiring laser treatment [24]. An earlier Turkish retrospective cohort study observed that $30.2 \%$ of ELBW infants who had advanced ROP required laser treatment [26]. Fortes et al. reported that $48.9 \%$ of ELBW infants in southern Brazil developed threshold ROP requiring laser treatment [19]; however, a study conducted in China [31] reported that the incidence of ROP requiring laser treatment was $28.6 \%$, in contrast to $28.7 \%$ in a study of ELBW infants conducted in the USA [32]. A Canadian study reported that the overall incidence of stage $\geq 3$ ROP in ELBW infants was $17.3 \%$ [6]. The lowest incidence of ROP requiring laser treatment in ELBW infants was reported from Brazil (12.7\%) [33]. The variation in the reported incidence of severe ROP in the literature is due to numerous factors such as low birth weight (BW), low gestational age (GA), sex, presence of respiratory distress syndrome (RDS), presence of blood culture-proven sepsis, presence of necrotizing enterocolitis (NEC), presence of intraventricular hemorrhage (IVH), and presence of patent ductus arteriosus (PDA) [34].
Also, we think the high incidence of severe ROP in the present study was primarily due to the fact it was conducted in a tertiary referral center that treats ROP patients requiring laser treatment from all over Turkey, and that most of the patients that admitted to our clinic were sick infants and at high-risk for developing severe ROP.

In the present study, low gestational age at birth was a significant risk factor for developing any stage of ROP, according to univariate analysis. Mean gestational age at birth was $28.34 \pm 1.91$ weeks in the infants without ROP vs. $26.65 \pm 1.84$ weeks in those with ROP. When the patients in the present study were divided into 2 subgroups according to gestational age at birth ( $\leq 25$ weeks vs. $\geq 26$ weeks), $90 \%$ (45/50) of the ELBW infants delivered at $\leq 25$ weeks developed ROP vs. $88 \%$ in a study by Isaza et al. [6] and $87 \%$ in a study by Teed et al. [35]. These similar percentages are in agreement with the general knowledge that prematurity plays an important role in the pathogenesis of ROP [34,36-42]. In the present study, the severity of ROP was inversely related to gestational age at birth $(P=0.008)$ and gestational weight at birth $(P=0.011)$, based on univariate and multivariate logistic regression analyses. Because gestational age at birth decreased, the duration of assisted ventilation and the likelihood of development of severe ROP increased [43]. Shah et al. reported that extreme low birth weight $(<1000 \mathrm{~g})$ and extreme prematurity $(<30$ weeks) were the main risk factors for developing severe ROP [44], and Fortes et al. reported that one of the main risk factors for severe ROP in their retrospective ELBW infant cohort was gestational age at birth [33]; the present findings are in agreement with these earlier findings.

Univariate analysis and multivariate logistic regression analysis showed that various systemic risk factors, such as the presence of culture-proven sepsis and blood transfusion, were strongly correlated with the development of severe ROP in the present study. Previous reports on very low birth weight infants reported $\geq 1$ of these risk factors, but the present study is the first to 
report the pathogenic role of these factors in ELBW infants; clinicians must be aware of the importance of these risk factors in ELBW infants and that they can result in severe ROP [44].

Araz et al. reported that there was a strong association between the presence of ROP (as well as severe ROP) and sepsis [45]. Weintraub et al. noted that sepsis increases the risk of developing ROP 12-fold; they hypothesized that sepsis might increase oxygen demand and interfere with oxygen tension, which might increase retinal ischemia, resulting in ROP [46]. In the present study sepsis was an important and significant predictor of developing severe ROP.

Blood transfusion was the most important significant associated risk factor for developing severe ROP in the present study. Earlier studies have reported that blood transfusion is a significant risk factor for the development of threshold disease $[42,47]$. Weintraub et al. reported that blood transfusion may be considered a clinical marker for the development of stage $\geq 3$ ROP; they reported that the incidence of stage 3 ROP was 14-fold higher in their neonates that received blood

\section{References:}

1. Smith LE: Pathogenesis of retinopathy of prematurity. Growth Horm IGF Res, 2004; 14: 140-44

2. Gilbert C: Retinopathy of prematurity: a global perspective of the epidemics, population of babies at risk and implications for control. Early Hum Dev, 2008; 84: 77-82

3. Valentine PH, Jackson JC, Kalina RE, Woodrum DE: Increased survival of low birth weight infants: impact on the incidence of retinopathy of prematurity. Pediatrics, 1989; 84: 442-45

4. Bullard SR, Donahue SP, Feman SS et al: The decreasing incidence and severity of retinopathy of prematurity. J AAPOS, 1999; 3: 46-52

5. Chiang MF, Arons RR, Flynn JT, Starren JB: Incidence of retinopathy of prematurity from 1996 to 2000: analysis of a comprehensive New York state patient database. Ophthalmology, 2004; 111: 1317-25

6. Isaza G, Arora S: Incidence and severity of retinopathy of prematurity in extremely premature infants. Can J Ophthalmol, 2012; 47: 296-300

7. Ebrahim M, Ahmad RS, Mohammad M: Incidence and risk factors of retinopathy of prematurity in Babol, North of Iran. Ophthalmic Epidemiol, 2010; 17: $166-70$

8. Fortes Filho JB, Eckert GU et al: The influence of gestational age on the dynamic behavior of other risk factors associated with retinopathy of prematurity (ROP). Graefes Arch Clin Exp Ophthalmol, 2010; 248: 893-900

9. Wani VB, Kumar N, Sabti K et al: Results of screening for retinopathy of prematurity in a large nursery in Kuwait: Incidence and risk factors. Indian J Ophthalmol, 2010; 58: 204-8

10. International Committee for the Classification of Retinopathy of Prematurity. The International Classification of Retinopathy of Prematurity revisited. Arch Ophthalmol, 2005; 123: 991-99

11. Fierson WM, American Academy of Pediatrics Section on Ophthalmology, American Academy of Ophthalmology, American Association for Pediatric Ophthalmology and Strabismus, American Association of Certified Orthoptists: Screening examination of premature infants for retinopathy of prematurity. Pediatrics, 2013; 131: 189-95

12. Early Treatment for Retinopathy Of Prematurity Cooperative Group: Revised indications for the treatment of retinopathy of prematurity: results of the early treatment for retinopathy of prematurity randomized trial. Arch Ophthalmol, 2003; 121: 1684-94

13. Markestad T, Kaaresen PI, Ronnestad A et al: Early death, morbidity, and need of treatment among extremely premature infants. Pediatrics, 2005 115: $1289-98$ transfusions, and posited that it might have been because the high oxygen concentration of the transfused blood may have had toxic effects on the immature peripheral retinal vessels. They also found that a reduction in blood transfusions led to a reduction the incidence of severe ROP [46].

\section{Conclusions}

In the present study, the severity of ROP was inversely correlated with birth weight and gestational age at birth, and positively correlated with culture-proven sepsis and blood transfusion. Clinicians should be aware of the presence of these risk factors when treating premature infants. Early detection and prevention of sepsis and reducing the number of blood transfusions may decrease the incidence of severe ROP requiring laser treatment. The identification of other risk factors that might be associated with the severity of ROP warrants further investigation. Additional analysis of the risk factors for severe ROP will improve our understanding and ability to predict the development of severe ROP in extremely premature infants.

14. Ells A, Hicks $M$, Fielden $M$, Ingram A: Severe retinopathy of prematurity: longitudinal observation of disease and screening implications. Eye, 2005; 19: $138-44$

15. Good WV, Hardy RJ, Dobson V et al: The incidence and course of retinopathy of prematurity: findings from the early treatment for retinopathy of prematurity study. Pediatrics, 2005; 116: 15-23

16. Fielder AR: Time for a fresh look at ROP screening. Eye, 2003; 17: 117-18

17. Reynolds JD, Dobson V, Quinn GE et al: Evidence-based screening criteria for retinopathy of prematurity: natural history data from the CRYO-ROP and LIGHT-ROP studies. Arch Ophthalmol, 2002; 120: 1470-76

18. Austeng D, Kallen KB, Ewald UW et al: Incidence of retinopathy of prematurity in infants born before 27 weeks' gestation in Sweden. Arch Ophthalmol, 2009; 127: 1315-19

19. Fortes Filho JB, Eckert GU, Procianoy L et al: Incidence and risk factors for retinopathy of prematurity in very low and in extremely low birth weight infants in a unit-based approach in southern Brazil. Eye, 2009; 23: 25-30

20. Cryotherapy for Retinopathy of Prematurity Cooperative Group: Multicente trial of cryotherapy for retinopathy of prematurity. Preliminary results. Arch Ophthalmol, 1988; 106: 471-79

21. Kumar P, Sankar MJ, Deorari A et al: Risk factors for severe retinopathy of prematurity in preterm low birth weight neonates. Indian J Pediatr, 2011; 78: 812-16

22. Lorenz B, Spasovska K, Elflein $\mathrm{H}$, Schneider N: Wide-field digital imaging based telemedicine for screening for acute retinopathy of prematurity (ROP). Six-year results of a multicentre field study. Graefes Arch Clin Exp Ophthalmol, 2009; 247: 1251-62

23. Zin AA, Moreira ME, Bunce $C$ et al: Retinopathy of prematurity in 7 neonatal units in Rio de Janeiro: screening criteria and workload implications. Pediatrics, 2010; 126: 410-17

24. Choo MM, Martin FJ, Theam LC, U-Theng C: Retinopathy of prematurity in extremely low birth weight infants in Malaysia. J AAPOS, 2009; 13: 446-49

25. Li Q, Yin ZQ, Chen L et al: Incidence of Retinopathy of Prematurity in Southwestern China and Analysis of Risk Factors. Med Sci Monit, 2014 20: 1442-51

26. Demir S, Sayin O, Aygun C et al: Retinopathy of prematurity in extremely low birth weight infants in Turkey. J Pediatr Ophthalmol Strabismus, 2013; 50: 229-33 
27. Alpay A, Ugurbas SH: Incidence and risk factors for retinopathy of prematurity in the West Black Sea region, Turkey. Turk J Pediatr, 2012; 54: 113-18

28. Hiraoka M, Watanabe T, Kawakami T et al: Retinopathy of prematurity in extremely low birth weight infants: a Tokyo multicenter study. Nihon Ganka Gakkai Zasshi, 2004; 108: 600-5

29. Gharaibeh A, Khassawneh M, Khriesat W et al: Adopting Western Retinopathy of Prematurity Screening Programs in Eastern Countries, are we Screening Properly? Middle East Afr J Ophthalmol, 2011; 18: 209-13

30. Wani VB, Uboweja AK, Gani M et al: Type I retinopathy of prematurity in infants with birth weight less than $1251 \mathrm{~g}$ : incidence and risk factors for its development in a nursery in Kuwait. Middle East Afr J Ophthalmol, 2013; 20: $66-71$

31. Chen $\mathrm{Y}$, Li XX, Yin $\mathrm{H}$ et al: Risk factors for retinopathy of prematurity in six neonatal intensive care units in Beijing, China. Br J Ophthalmol, 2008; 92: 326-30

32. Lad EM, Hernandez-Boussard T, Morton JM, Moshfeghi DM: Incidence of retinopathy of prematurity in the United States: 1997 through 2005. Am J Ophthalmol, 2009; 148: 451-58

33. Fortes Filho JB, Borges Fortes BG, Tartarella MB, Procianoy RS: Incidence and Main Risk Factors for Severe Retinopathy of Prematurity in Infants Weighing Less Than 1000 Grams in Brazil. J Trop Pediatr, 2013; 59: 502-6

34. Darlow BA, Hutchinson JL, Simpson JM et al: Variation in rates of severe retinopathy of prematurity among neonatal intensive care units in the Australian and New Zealand Neonatal Network. Br J Ophthalmol, 2005; 89: 1592-96

35. Teed RG, Saunders RA: Retinopathy of prematurity in extremely premature infants. J AAPOS, 2009; 13: 370-73

36. Dammann O: Inflammation and retinopathy of prematurity. Acta Paediatr, 2010; 99: 975-77
37. Dammann O, Brinkhaus MJ, Bartels DB et al: Immaturity, perinatal inflammation, and retinopathy of prematurity: a multi-hit hypothesis. Early Hum Dev, 2009; 85: 325-29

38. Hussain N, Clive J, Bhandari V: Current incidence of retinopathy of prematurity, 1989-1997. Pediatrics, 1999; 104: e26

39. Brown BA, Thach AB, Song JC et al: Retinopathy of prematurity: evaluation of risk factors. Int Ophthalmol, 1998; 22: 279-83

40. Seiberth V, Linderkamp O: Risk factors in retinopathy of prematurity. a multivariate statistical analysis. Ophthalmologica, 2000; 214: 131-35

41. Bassiouny MR: Risk factors associated with retinopathy of prematurity: a study from Oman. J Trop Pediatr, 1996; 42: 355-58

42. Akkoyun I, Oto S, Yilmaz G et al: Risk factors in the development of mild and severe retinopathy of prematurity. J AAPOS, 2006; 10: 449-53

43. Allegaert K, Vanhole C, Casteels I et al: Perinatal growth characteristics and associated risk of developing threshold retinopathy of prematurity. J AAPOS, 2003; 7: 34-37

44. Shah VA, Yeo CL, Ling YL, Ho LY: Incidence, risk factors of retinopathy of prematurity among very low birth weight infants in Singapore. Ann Acad Med Singapore, 2005; 34: 169-78

45. Araz-Ersan B, Kir N, Akarcay K et al: Epidemiological analysis of retinopathy of prematurity in a referral centre in Turkey. Br J Ophthalmol, 2013; 97: $15-17$

46. Weintraub Z, Carmi N, Elouti H, Rumelt S: The association between stage 3 or higher retinopathy of prematurity and other disorders of prematurity. Can J Ophthalmol, 2011; 46: 419-24

47. Allegaert K, de Coen K, Devlieger H, EpiBel Study Group: Threshold retinopathy at threshold of viability: the EpiBel study. Br J Ophthalmol, 2004; 88: 239-42 